\title{
INFECTIONS AND PREMATURITY, IMPORTANT RISK FACTORS FOR NEONATAL MORBIDITY AND MORTALITY
}

\author{
Elena Tarca ${ }^{1}$, Simona Gavrilescu ${ }^{1}$, Laura Florescu², Alina Mariela Murgu ${ }^{3}$, \\ Monica Ungureanu ${ }^{4}$, Vasile Valeriu Lupu ${ }^{3}$, Dana Elena Mindru ${ }^{2}$ \\ 1 "Grigore T. Popa" Department of Pediatric Surgery and Orthopedics, \\ University of Medicine and Pharmacy, Iasi, \\ 2 "Grigore T. Popa" Department of Infantcare, University of Medicine and Pharmacy, Iasi, \\ 3 "Grigore T. Popa" Department of Pediatrics, University of Medicine and Pharmacy, Iasi, \\ 4 "Grigore T. Popa" Department of Preventive Medicine and Interdisciplinarity, \\ University of Medicine and Pharmacy, Iasi,
}

\begin{abstract}
Infant mortality is a major problem in developing countries and, unfortunately, this is the case of our country as well, given that Romania ranks first in the European Union in this respect, with an infant mortality rate of $9 \%$, compared to an average of roughly $4 \%$. Worldwide, over 15 million babies are born prematurely each year and, out of these, more than a million die due to prematurity and infections, which are the main risk factors for neonatal mortality. The risk of infection is several times higher in preterm newborns than in full-term newborns - about $80 \%$ of neonatal infections occur in premature infants. A significant proportion of the survivors of prematurity will have important neurological sequelae because of neonatal infections as well as of intracerebral bleeding or hypoxia at birth. Continuing medical education in both the general population and the medical sector is crucial in preventing premature births and neonatal infections and, consequently, in decreasing infant morbidity and mortality rates in our country.
\end{abstract}

Keywords: neonatal infections, prematurity, morbidity, infant mortality

Globally, in recent years, the infant mortality rate (in the neonatal period) is $44 \%$ in children under 5 years of age and in $85 \%$ of cases death occurs due to hypoxia at birth, perinatal infections, prematurity or low birth weight (1). About 1 million of the 2.7 million deaths that occurred in the neonatal period in 2013 took place on the first day of the child's life; it was estimated that about two thirds of these deaths could have been avoided by quality perinatal care (2).

Over 15 million children are born prematurely (under 37 weeks of gestation) and, out of these, more than one million die, with prematurity as the main risk factor for neonatal mortality in recent decades, which affects over $11 \%$ of pregnancies (3). In Romania, in the last two decades, closer involvement of authorities as well as of health professionals in actions aimed at ensuring the survival of premature newborns had direct and visible effects, such as the decrease of the infant mortality rate from 24 deaths per thousand births in 1994 to 9\%o in 2012 and 8\%o in 2015 (4). However, infant mortality remains a major problem in our country, as Romania ranks first in the European Union in this respect, with an infant mortality rate of $8 \%$, compared to the average figure of under $4 \%$ (4). 
Another important cause of the neonatal mortality is infection, causing over 4,000,000 deaths annually and 3,000 deaths daily in neonates worldwide. The risk of infection is several times higher in preterm infants compared to full-term infants - approximately $80 \%$ of neonatal infections occur in premature infants (5). Infections in fetuses and newborns can be congenital, which means that they are acquired in utero by transplacental transmission. They can also be perinatal, that is acquired between 22 weeks of gestation and seven days after birth, but they can also be neonatal infections, such as those acquired immediately after birth and up to 28 days of life (4). Maternal-fetal infections can be caused by pathogens transmitted from mother, through transplacental transmission, contact with contaminated maternal blood or vaginal secretions, and they are microbiologically obvious (not necessary clinically - e.g. HIV) in newborns as early as the first 48 hours of life. Of the infections transmitted from the mother, most important are those of the TORCH syndrome, HIV, herpetic infection, hepatitis B and C, congenital syphilis (infection with Treponema pallidum). A special category of neonatal infections are healthcare-associated infections (HAI) or nosocomial infections, in which case the pathogens are contracted by healthy newborns from people around them or the environment (instrumentation and medical equipment) immediately after birth. They usually become manifest 3 days after birth, depending on the etiology and severity.

Neonatal sepsis is most commonly caused by Gram-positive germs - such as group B streptococcus (GBS), Streptococcus pneumoniae, Enterococcus spp. and coagulase-negative staphylococci (roughly 50-75\%), but the most serious infection is the one caused by Gram-negative germs like Escherichia coli (6-8). Early onset neonatal sepsis occurs within the first 24 hours of life and is a fulminant multisystem infection, acquired by vertical transmission from the mother. Risk factors for the early onset neonatal sepsis are: prematurity, maternal colonization with GBS, early rupture of the amniotic membranes, chorioamniotitis and intra-partum fever $(9,10)$. The incidence of early sepsis in full-term neonates is $1-2$ / 1000 , with a mortality of about $3 \%$. In terms of responsible pathogens, the most commonly involved are GBS (41\%) and Escherichia coli (17\%) (11). Premature infants and newborns with very low birth weight especially are at higher risk of developing early onset neonatal sepsis; more precisely, the risk is over 10 times higher for this categories; in their case, mortality also exceeds $30 \%$. The most commonly involved pathogens are Gram-negative germs (12).

Another significant proportion of the pediatric population at high risk of developing hospital-acquired infections are children undergoing surgery, for whom surgical wound infections (approximately 4-7\% of the patients operated on) are complications associated with a significant increase in costs and hospital stay, as well as in morbidity and mortality rates (13). Of all healthcare-associated infections, surgical wound infections constitute a proportion of $20 \%$ to $31 \%$ $(14,15)$. The Surgical Infection Prevention Project was launched in 2002 in developed countries and promotes the optimal use of perioperative antimicrobial prophylaxis in order to reduce the occurrence of surgical wound infections, by following three simple principles: the use of the type of antibiotic that is most appropriate for the surgery and for the patient particularities, at the same time taking into account the effective protocol for that particular condition, the administration of antimicrobial prophylaxis by parenteral route 60 minutes prior to surgery and antibiotic discontinuation 24 hours after surgery or 48 hours in the case of cardiac interventions (16). Most organizations and treatment guidelines discourage the prolonged use of antimicrobial prophylaxis, as this inadequate practice may lead to the appearance of microbial strains that 
are resistant to antibiotics, of infections with Candida spp. or Clostridium difficile associated infection (or disease), in addition to the toxicity itself of antibiotics and without the benefit of a significant decrease of the infections risk (17, 18). Since there are no generally accepted protocols for perioperative antimicrobial prophylaxis in children, treatment guidelines for adults are often used, namely: cefazolin or cefuroxime for cardiac surgery and vancomycin or clindamycin in case of allergy to beta-lactam antibiotics. Cefazolin is used for abdominal surgery without enterotomy, and in case of more complex surgery, cefotetan, cefoxitin, cefazolin + metronidazole can be used (17). In connection with hospital-acquired infections, there are special guidelines in the departments of neonatology and in intensive care units especially and these guidelines need to be followed in order to avoid the infections related to the venous approach and the central venous catheterization. Such infections lead to an increase in the duration and cost of hospitalization. The following are some of the most important recommendations among these guidelines (19): 1) continuing the medical education and training of the medical personnel on the insertion and maintenance of intravascular catheters; 2) the insertion of the central venous catheter will be carried out under aseptic and antiseptic conditions; 3 ) the disinfection of the skin requires the use of a solution of chlorhexidine having a concentration of $>0,5 \%$; 4 the strategy of preventing hospital-acquired infections does not include the routine replacement of central venous catheters; 5 ) if the rate of infection in a specific section does not decrease although previous recommendations have been followed, central venous catheters impregnated with antiseptic or antibiotic substances will be used and will be changed regularly and the dressings will be impregnated with a chlorhexidine solution (19). The risk factors for hospitalacquired infections in newborns, including those related to the central venous approach or to the surgical wound infections, are different from those in adults. Here we can mention prematurity and low birth weight, immune deficiency characteristic to newborns, immaturity of the skin barrier, the need for medical equipment use, such as the central venous catheter, associated malformations and prolonged hospitalization (13).

A significant proportion of the survivors of prematurity will remain with important neurological sequelae due to neonatal infections, intracerebral bleeding or hypoxia at birth (20). There are studies showing that any form of neonatal infection (sepsis with positive cultures, meningitis and ulceronecrotic enterocolitis with or without sepsis) is associated with poor somatic development and a high risk of neurological disorders $(20,21)$. More and more studies demonstrate that neonatal infections, even in the absence of microbial direct damages of the neurological structures, by triggering the (systemic) inflammatory response, can cause damages of the white matter with long-term neurological sequelae, particularly in former preterm newborns (20-23).

Approximately 50 million births $(40 \%$ of all births worldwide) occur outside the hospital environment in risky conditions for both the mother and the newborn (24). Following a few principles of basic neonatal care, such as giving birth in hygienic conditions, cutting the umbilical cord and applying antiseptic solutions on it, clearing the upper airways, keeping the newborn warm and encouraging breast-feeding, are simple but essential things for the survival of the newborn and, most of all, they are accessible in any environment where the birth might take place $(2,25)$.

Continuing medical education, both in the general population and in the medical sector, is crucial in preventing premature births and neonatal infections and, consequently, in decreasing infant morbidity and mortality rates. 


\section{REFERENCES}

1. Wang H., Liddell C.A., Coates M.M. et al. Global, regional, and national levels of neonatal, infant, and under-five mortality during 1990-2013: A systematic analysis for the Global Burden of Disease Study 2013. Lancet 2014, 384 (9947): 957-979.

2. Bhutta Z.A., Das J.K., Bahl R. et al. Every newborn: Can interventions end preventable deaths available in mothers, newborn babies, and stillbirths, and at what cost? Lancet 2014, 384 (9940): 347-370.

3. Blencowe H., Cousens S., Oestergaard M.Z. et al. National, regional, and worldwide estimates of preterm birth rates in the year 2010 with time trends since 1990 for selected countries: a systematic analysis and Implications. Lancet 2012, 379 (9832): 2162-72. [PubMed: 22682464]

4. http://www.ccss.ro/public_html/sites/default/files//MORTALITATEA\%20INFANTILA\%202015.pdf

5. Liu L., Johnson H.L., Cousens S. et al. Global, regional, and national causes of child mortality: an updated systematic analysis with time trends for 2010 since 2000. Lancet 2012, 379 (9832): 2151-61.

6. Simonsen K.A., Anderson-Berry A.L., Delair S.F. et al. Early-Onset Neonatal Sepsis. Clin Microbiol Rev 2014 Jan; 27(1): 21-47. doi: 10.1128/CMR.00031-13

7. Hoffman J.A., Mason E.O., Schutze G.E. et al. Streptococcus pneumoniae infections in the neonate. Pediatrics 2003, 112:10951102. 10.1542/peds.112.5.1095

8. Bizzarro M.J., Raskind C., Baltimore R.S. et al. Seventy-five years of neonatal sepsis at Yale: 1928-2003. Pediatrics 2005, 116:595-602. 10.1542/peds.2005-0552

9. Benitz W.E., Gould J.B., Druzina M.L. Risk factors for early-onset group B streptococcal sepsis: estimation of odds ratios by critical literature review. Pediatrics, Jun, 1999, 103 (6): E77. [PubMed: 10353974]

10. Gebremedhin D., Berhe H., Gebrekirstos K. Risk Factors for Neonatal Sepsis in Public Hospitals of Mekelle City, North Ethiopia, 2015: Unmatched Case Control Study. PLoS One 2016, 11(5): e0154798. Published online 2016 May 10. doi: 10.1371/journal. pone.0154798

11. Hyde T.B., Hilger T.M., Reingold A. et al. Trends in incidence and antimicrobial resistance of early-onset sepsis: Population-based surveillance in San Francisco and Atlanta. Pediatrics, October, 2002, 110 (4): 690-695. [PubMed: 12359781]

12. Stoll B.J., Hansen N.I., Higgins R.D. et al. Very low birth weight preterm Infants with neonatal sepsis early onset: the predominance of gram-negative Infections Continues in the National Institute of Child Health and Human Development Neonatal Research Network, 2002-2003. Pediatr Infect Dis J, Jul; 2005, 24 (7): 635-639. [PubMed: 15999007]
13. Prasad A.P., Wong-McLoughlin J., Patel S. et al. Surgical Site Infections in a Longitudinal Cohort of Patients Neonatal Intensive Care Unit. J Perinatol, April 2016, 36 (4): 300-305. doi: 10.1038 / jp.2015.191.

14. Lissovoy G., Fraeman K., Hutchins V. et al. Surgical site infection: Incidence and impact on hospital utilization and treatment costs. Am J Infect Control 2009, 37: 387-397. [PubMed: 19398246]

15. Magill S.S., Hellinger W., Cohen J., et al. Prevalence of healthcareassociated Infections in Acute Hospitals in Jacksonville, Florida. Infect Control Hosp Epidemiol 2012, 33 (3): 283-291. [PubMed: 22314066]

16. Bratzler D.W., Houck P.M. Antimicrobial prophylaxis for surgery: an advisory statement from the National Surgical Infection Prevention Project. Clin Infect Dis 2004, 38 (12): 1706-1715. [PubMed: 15227616]

17. Bratzler D.W., Dellinger E.P., Olsen K.M. et al. Clinical practice guidelines for antimicrobial prophylaxis in surgery. Am J Health Syst Pharm 2013, 70: 195-283. [PubMed: 23327981]

18. Edwards F.H., Engelman R.M., Houck P. et al. The Society of Thoracic Surgeons practice guideline series: antibiotic prophylaxis in cardiac surgery, Part I: Duration. Ann Thorac Surg 2006, 81 (1): 397-404. [PubMed: 16368422]

19. O'Grady N.P., Alexander M., Burns L.A. et al. Guidelines for the Prevention of Intravascular Catheter-Related Infections. Clin Infect Dis 2011, May 1; 52 (9): e162-e193. doi: 10.1093 / cid / cir257.

20. Johnson S., Fawke J., Hennessy E. et al. Neurodevelopmental disability through 11 years of age in children born before 26 weeks of gestation. Pediatrics 2009, 124:e249-57.

21. Stoll B.J., Hansen N.I., Adams-Chapman I. et al. Neurodevelopmental and growth impairment among extremely low-birth-weight Infants with neonatal infection. JAMA. 2004, 292 (19): 2357-65. [PubMed: 15547163]

22. Strunk T., Inder T., Wang X. et al. Infection and Inflammation-Induced Cerebral Injury in Preterm Infants. Lancet Infect Dis August 2014, 14 (8): 751-762.

23. Wood N.S., Marlow N., Costeloe K. et al. Neurologic and developmental disability after extremely preterm birth. EPICure Study Group. N Engl J Med 2000, 343:378-84.

24. Lawn J.E., Blencowe H., Oza S. et al. Every newborn: Progress, priorities, and potentially beyond survival. Lancet 2014, 384 (9938): 189-205.

25. Enweronu-Laryea C., Dickson K.E., Moxon S.G. et al. Basic newborn care and neonatal resuscitation: A multi-country analysis of health system bottlenecks and potential solutions. BMC Pregnancy and Childbirth 2015, 15 (Suppl 2): S4. 